\title{
BMJ Open International survey for assessing COVID-19's impact on fear and health: study protocol
} Kris Yuet-Wan Lok (D) , Daniel Yee Tak Fong (D) , Janet Y.H. Wong (D) , ${ }^{1}$
Mandy Ho (D) ,' Edmond PH Choi, ${ }^{1}$ Vinciya Pandian, ${ }^{2}$ Patricia M Davidson, ${ }^{2}$
Wenjie Duan, ${ }^{3}$ Marie Tarrant, ${ }^{4}$ Jung Jae Lee, ${ }^{1}$ Chia-Chin Lin, ${ }^{1}$ CARE group

To cite: Lok KY-W, Fong DYT, Wong JY.H, et al. International survey for assessing COVID19's impact on fear and health: study protocol. BMJ Open 2021;11:e048720. doi:10.1136/ bmjopen-2021-048720

- Prepublication history and additional online supplemental material for this paper are available online. To view these files, please visit the journal online (http://dx.doi.org/10. 1136/bmjopen-2021-048720).

Received 05 January 2021 Revised 20 April 2021 Accepted 29 April 2021
Check for updates

(c) Author(s) (or their employer(s)) 2021. Re-use permitted under CC BY-NC. No commercial re-use. See rights and permissions. Published by BMJ.

${ }^{1}$ School of Nursing, University of Hong Kong Li Ka Shing Faculty of Medicine, Hong Kong, Hong Kong

${ }^{2}$ School of Nursing, Johns Hopkins University, Baltimore, Maryland, USA

${ }^{3}$ Department of Social Work, East China University of Science and Technology, Shanghai, China ${ }^{4}$ School of Nursing, The University of British Columbia, Vancouver, British Columbia, Canada

Correspondence to Dr Daniel Yee Tak Fong; dytfong@hku.hk

\section{ABSTRACT}

Introduction COVID-19, caused by the SARS-CoV-2, has been one of the most highly contagious and rapidly spreading virus outbreak. The pandemic not only has catastrophic impacts on physical health and economy around the world, but also the psychological well-being of individuals, communities and society. The psychological and social impacts of the COVID-19 pandemic internationally have not been well described. There is a lack of international study assessing health-related impacts of the COVID-19 pandemic, especially on the degree to which individuals are fearful of the pandemic. Therefore, this study aims to (1) assess the health-related impact of the COVID-19 pandemic in community-dwelling individuals around the world; (2) determine the extent various communities are fearful of COVID-19 and (3) identify perceived needs of the population to prepare for potential future pandemics.

Methods and analysis This global study involves 30 countries. For each country, we target at least 500 subjects aged 18 years or above. The questionnaires will be available online and in local languages. The questionnaires include assessment of the health impacts of COVID-19, perceived importance of future preparation for the pandemic, fear, lifestyles, sociodemographics, COVID-19-related knowledge, e-health literacy, out-ofcontrol scale and the Patient Health Questionnaire-4. Descriptive statistics will be used to describe participants' characteristics, perceptions on the health-related impacts of COVID-19, fear, anxiety and depression, lifestyles, COVID-19 knowledge, e-health literacy and other measures. Univariable and multivariable regression models will be used to assess the associations of covariates on the outcomes.

Ethics and dissemination The study has been reviewed and approved by the local ethics committees in participating countries, where local ethics approval is needed. The results will be actively disseminated. This study aims to map an international perspective and comparison for future preparation in a pandemic.

\section{BACKGROUND}

COVID-19, caused by the SARS-CoV-2, has precipitated a global pandemic causing death, disability and economic devastation globally. As of 25 November 2020, there have been

\section{Strengths and limitations of this study}

- This study examines the global the impact of COVID-19 pandemic on lifestyle behaviours, fear, depression and perceived needs of communities.

- The survey will be administered in 30 countries across the globe with varying cultural perspectives towards COVID-19.

- Individuals with low socioeconomic status, no digital literacy and insufficient access to a digital device or the internet might be under-represented.

- The cross-sectional design of the study might limit the exploration of longitudinal changes within each community.

over 60 million confirmed cases of COVID-19 worldwide. ${ }^{1}$ In a WHO brief published in August 2020, the infection-fatality ratio is estimated to be around $0.5 \%-1 \%{ }^{2}$ The USA recorded the highest number of cases, with $20.6 \%$ of total global cases, followed by India $(18.5 \%)$, Brazil (12.7\%), Russia (3.5\%), Spain $(2.6 \%)$ and Argentina $(2.5 \%) .{ }^{1}$ In less than a year, the COVID-19 pandemic has caused unprecedented disruptions in healthcare systems and the global economy. Pandemics and plagues have informed the narrative of many nations. ${ }^{3}$ Previous pandemics have underscored the importance of considering fear, information management and its impact on health behaviours.

Although the roll-out of several effective vaccines revealed a potential path towards the end of the COVID-19 pandemic, the emergence of new variants and the logistical challenges in making vaccines available to middle-income and lower-income countries likely mean that the current pandemic will continue to affect the global population in the coming months. In the meantime, extensive and strict public health measures have been put into effect to minimise the spread of COVID-19. At the policy level, countries 
have implemented measures such as travel restrictions in severely affected areas, ceasing or limiting transportation, restricting the hours or closing restaurants and businesses with a high risk of virus transmission, imposing mass quarantine or lockdown, and other measures such as mandatory face-covering in public spaces. ${ }^{4}$ At an individual level, the public is recommended to adopt preventative measures including social distancing, avoiding crowds and indoor gatherings, and practising good personal hygiene, like handwashing and wearing face masks. ${ }^{45}$

Coupled with the threat of COVID-19, these abrupt changes have a profound impact on individuals in terms of health and other health-related issues. During isolation and quarantine, adjustments in health behaviours, including changes in eating habits and physical activities have been observed. ${ }^{67}$ It is suggested that pandemicrelated stress and lifestyle changes may contribute to increased cardiovascular risk. ${ }^{8}$ A study has found worsening of symptoms in individuals with Parkinson's disease during a lockdown. ${ }^{9}$ In addition, there was increasing evidence that fear of contracting COVID-19 made patients avoid visiting emergency units and lead to increased mortality. ${ }^{10}$

The restrictive public health measures and fear associated with the pandemic could also impact an individual's mental well-being. At the early stage of the pandemic, several studies have been conducted to examine the psychological and social impacts of COVID-19. Studies have reported increased level of stress associated with national lockdown. ${ }^{11}$ Policies such as social distancing, use of face masks and returning to work have been also been found to be related to varying level of stress and anxiety. ${ }^{12-14}$ A study in a Chinese province found that the pandemic had a mild stressful effect on individuals but also reported the presence of increased social support and increased social cohesion. ${ }^{15}$ A later longitudinal study in China reported that levels of stress, anxiety and depression in the general population remain stable. ${ }^{16} \mathrm{~A}$ study focused the Diamond Princess Cruise has found deteriorated quality of life in passengers and crew members during quarantine, with fear of getting infected and the lack of medical care on the cruise being key factors. ${ }^{17}$

More recent studies have reported higher levels of emotional distress and increased suicidal ideation in young adults during the pandemic, ${ }^{18} 19$ elevated depressive and anxiety symptoms in younger adults during stayat-home order, ${ }^{20}$ and higher loneliness, and depression in older adults during shelter-in-place order. ${ }^{21} \mathrm{~A}$ longitudinal study in Austria has found that higher stress and loneliness during lockdown are associated with depression afterwards. ${ }^{22}$ Studies in Vietnam and Philippines have also found increased anxiety and stress in patients with psychiatric disorders and illnesses such as systemic lupus erythematosus and rheumatoid arthritis during lockdown. ${ }^{23}$ Additionally, studies focusing on healthcare workers have found that they reported depression, anxiety, post-traumatic stress disorder and stress symptoms amid the COVID-19 outbreak. ${ }^{25-27}$
These findings have shown that the COVID-19 pandemic and public health measures intended to limit its spread could have a detrimental impact on the population's mental health. A recent review examining the mental health impact of quarantine measures from previous epidemics has identified fear of infection, inadequate basic supplies, insufficient information, frustration and boredom as major stressors during quarantine, and financial pressure as a key stressor postquarantine. ${ }^{28}$ Moreover, the flood of COVID-19 information from official or unofficial social media platforms might also create uncertainty and anxiety in the general public. $^{29}$

Existing studies provided valuable insight into some of the pandemic's impact on the health-related issues on individuals; however, there is still a lack of studies on how the impacts differ across countries. Additionally, the current literature has focused mainly on the short-term psychological impact of the pandemic. To our knowledge to date, there has only been one multinational study conducted on the health impact of COVID-19 in Asia. ${ }^{16}$ The study has identified important risk factors, including age, educational background and marital status, and found cross-country differences in which countries with lowest rate of infection seem to show lowest levels of depression, anxiety and stress. ${ }^{16}$ While the study provide important insight into the impact of COVID-19 of the pandemic on the physical and mental health of individuals, its focus was on seven middle-income countries in Asia and there is a lack of investigation on the impact of the pandemic responses. ${ }^{16}$ There remains a need for a global collaborative effort in assessing health-related impacts of COVID-19, especially on the degree to which individuals are fearful of the pandemic. How the varying severity of the pandemic and different preventive measures adopted across cities or communities in different parts of the world influence the health of the population remains to be examined.

With communities worldwide having had a complete cycle of the pandemic, it is timely to assess how it may have influenced our health and health-related issues. It could provide valuable information to help us understand the impacts of the pandemic more fully. It could also help to determine areas in which support would be needed and inform the development of recovery initiatives and clinical response where necessary. On top of that, the current pandemic has highlighted an urgent need for improving pandemic preparedness. While the pandemic measures were necessary in curbing the spread of the virus, they were often devised and implemented without much knowledge into their impacts on individuals' mental and physical health. The current study could provide much needed information on the impact of the pandemic and the different public health responses in different countries and help inform policies in preparation for and response to future virus outbreak. 


\section{METHODS AND ANALYSIS}

\section{Study aims}

This study aims to (1) assess the health-related impact of the COVID-19 pandemic in community-dwelling individuals around the world, (2) determine the extent the various communities are fearful of COVID-19 and (3) identify future needs in the preparation for a pandemic.

\section{Study design}

This study will use a cross-sectional design with questionnaires available in 10 languages and 30 participating countries to date. The questionnaires will be made available online through 'COVID-19's impact on fear and health (CARE)' International Survey (https://care.hku. hk), or other electronic platforms deemed appropriate in the individual local settings.

The Traditional Chinese version of the survey was the first rolled out. It was piloted on 20 June 2020, with data collection started on 3 July 2020 . The data analysis would be conducted in June 2021.

\section{Subjects and sampling}

We seek to enrol 500 participants aged 18 years or above, in each country. Participants will be recruited by survey service providers, social media or/and snowball sampling, whichever is/are feasible in the country (see online supplemental material). Specifically, survey service providers will contact subjects randomly drawn from their databases of participants who had previously taken part in their other studies and had consented to being contacted for future studies. Moreover, we shall advertise on social media platforms, for example, Facebook, WeChat, Twitter and LinkedIn. In addition, we will also reach subjects at universities, health clinics, and community centres by using posters and/or emails and invite them to disseminate our survey to their friends and family members. To enhance the participation rate ${ }^{30}$ for each completed questionnaire, HK\$1 (equivalently, US\$0.13) will be donated to the Red Cross in the respondent's region.

The sample size calculation was based on estimating the prevalence of a health-related issue. Taking the most conservative scenario of $50 \%$, with a $5 \%$ margin of error in a $95 \%$ CI, we need 385 subjects in a country. Taking incomplete questionnaires into consideration, we will target to get at least 500 subjects in each participating country.

\section{Measurements}

\section{Questionnaire}

The questionnaire will be self-administered online to measure the health-related impacts of COVID-19, fear of COVID-19 and identify future needs or preparation for a pandemic. The proposed survey shall offer a comprehensive assessment of the impact of COVID-19 as well as the corresponding perceived needs for our community to live better in such a pandemic. In addition, our international survey enables cross-country comparisons. The development, translation, validation and description of the questionnaire are discussed.

\section{Development}

The questionnaire began with an extensive literature search on the health-related impact of COVID-19. Several discussion meetings were conducted with a team of researchers, comprising public health professionals, nurses and nutritionists in Hong Kong, where the potential survey items were populated and placed into nine categories: (1) assessment of the health impact of COVID-19, (2) perceived importance of future preparation for the pandemic, (3) fear, (4) lifestyles, (5) sociodemographics, (6) COVID-19-related knowledge, (7) eHealth literacy and (8) the Patient Health Questionnaire-4 (PHQ) item. Thereby, a preliminary questionnaire in English was derived.

The preliminary questionnaire was then further reviewed and discussed to ensure that the questions adequately met the study's aim, that is, confirming the face validity. Moreover, the clarity of the questions with appropriate response items was checked, and that any duplications or coinciding themes were removed. The flow of the items was also reviewed to facilitate the completion of the questionnaire.

In addition, questionnaire items will be discussed with experts in specific countries to examine the cultural acceptability and other relevant influences of COVID-19. An out-of-control scale will be added to assess the sense of out-of-control of individuals during the pandemic. Since there had not been a scale that assessed the perceived level of out of control in community dwelling individuals, the scale was developed by an academic social worker based on his experience of online services offered to over 200 community-dwelling individuals in Wuhan city who perceived a loss of control during lockdown. A total of 14 questions were first developed which were then reviewed by an expert panel comprising the developer and an academic statistician experienced in cultural adaptation. As a result, the 10-item out-of-control scale was derived. Refinement of items will be made for the derivation of the final version of the CARE questionnaire.

\section{Translation}

There are four multi-item instruments adopted in the CARE questionnaire: Fear Scale, eHealth Literacy Scale (eHEALS), Patient Health Questionnaire (PHQ-4) and Out-of-Control Scale. When any of them is not available in a language, standard forward-backward translation will be conducted. ${ }^{31}$ Specifically, for each translation, two bilingual persons with at least native-level fluency in the target language will conduct forward translation independently. A meeting with two forward translators chaired by another bilingual person will be held to work out a consensus version in the target language. If there is only one out of the three aforementioned persons native in the target language, the fourth person who is native in the target language will be invited to read the 
consensus version. The consensus version will then be back-translated into the original language. The developer or a person native in the original language will compare the backward and original versions. Discrepancies will be identified and revisions of the consensus version will be made. Thereby, we will obtain a semifinal version. The semifinal version will be assessed in five subjects who are at least 18 years of age and can read the target language. After informed consent, they will self-complete the semifinal version and then debrief using a structured form regarding the clarity and relevance of items in the questionnaire. Revisions will be made, and the final version in the target language will be developed.

The PHQ-4 does not require a license before it can be used or translated. For Fear Scale, eHEALS and Out-ofcontrol Scale, prior permission from the corresponding developers has been sought.

\section{Validation}

To enhance internal validity, we have incorporated the validation question: 'Where does the sun rise every day?' Moreover, before the CARE questionnaire in a language or an electronic survey platform is rolled out, the questionnaire will be pilot-tested in at least 10 subjects in each language. Pilot data will be reviewed to ensure data consistency across countries, and an adequate understanding of the items in the local groups is achieved.

For instruments that have not been psychometrically evaluated, we shall examine their internal reliability using the Cronbach's alpha, convergent validity by examining their association with variables that are known to be associated with the corresponding constructs, and factorial validity by conducting exploratory factor analysis. In addition, we will use the item response theory (IRT) to examine their reliability and validity, ${ }^{32}$ as well as their cross-cultural comparisons via differential item functioning assessment. ${ }^{33}$ The IRT approach is adopted instead of the conventional approaches because it is not sample-dependent.

\section{Description}

The final questionnaire consisted 11 sections: (1) sociodemographic characteristics, (2) COVID-19-related knowledge, (3) COVID-19 status, (4) lifestyle, (5) fear factor, (6) impact of the COVID-19 pandemic, (7) future preparation, (8) Fear Scale, (9) eHEALS, (10) PHQ-4 and (11) Out-of-control Scale. The questionnaire examines the key outcomes and covariates outlined in the previous section.

\section{Outcomes}

There are seven key outcome measures pertaining to the health-related impact of COVID-19, sense of control, perceived importance of possible preparation during a pandemic, lifestyles and fear associated with COVID-19. The following instruments are administered as part of the test battery.
Health-related impact of COVID-19

Participants will be asked to rate their health-related issues during the COVID-19 as compared with their normal life before the pandemic on a 5-point Likert scale. The health-related issues comprise physical and psychological well-being, dietary, exercise, sedentary behaviours and finance.

Perceived importance for possible preparation during a pandemic Participants will be asked to rate, on a 5-point Likert scale, their perceived importance of a list of items on the possible preparation during a pandemic. Examples of such items are online doctor consultation, instant personalised health advice by an online chatbot, online shopping, food delivery and getting medicine prescribed in a hospital visit/follow-up in a community pharmacy.

\section{Fear associated with diseases and adverse events}

Participants will be asked to rate, on a scale from 0 to 10 , their level of fear associated with diseases and adverse events, such as COVID-19, cancer, traffic accident, lack of social life, loss of family members, etc. A higher score indicates a higher level of fear associated with the specific disease or situation. The fear factor scale allow reference for interpreting the fear level of COVID-19 in relation to other diseases and adverse events.

\section{Fear Scale}

The 8-item Fear Scale was developed specifically to assess the fear level related to COVID-19. ${ }^{34}$ Each item will be rated on a 5-point Likert scale. The total score describes the fear level with a higher score indicating a higher fear level. ${ }^{34}$

\section{Anxiety and depression}

The 4-item PHQ-4 will be used to assess anxiety and depression. It has been validated in the general population. ${ }^{35}$ It comprises four items, each rated on a 4-point Likert scale. Two items will be used to assess anxiety, and another two will be used to assess depression. A higher score indicates the presence of severe anxiety or increased depressive symptoms.

\section{Lifestyles}

Participants' lifestyle during the COVID-19 pandemic will be assessed. The alcohol consumption and smoking habits of participants will be recorded, along with the duration of screen time, frequency of vigorous and moderate physical activities, and hours of sitting (being sedentary).

\section{The Out-of-Control Scale}

The 10-item Out-of-Control Scale was a self-developed instrument to assess participants' perceived level of loss of control during the COVID-19 pandemic. Each item is rated on a 6-point Likert Scale. A higher total scale indicates a higher perceived degree of out of control. The Scale was originally developed in Simplified Chinese, and had been translated into the local languages of the participating countries, where needed. 


\section{Covariates}

In addition to the outcome variables, five covariate measures will be assessed, including sociodemographic information, diagnosed medical history, perceived COVID-19 knowledge, COVID-19 status and eHealth literacy.

Sociodemographic characteristics include age, gender, marital status, occupational status, perceived social status, ${ }^{36}{ }^{37}$ pregnancy status (if applicable) and household size. Participants will be asked to report any existing medical diagnosis. COVID-19-related knowledge and susceptibility will be assessed using six items from the WHO behavioural survey on COVID-19, ${ }^{38}$ with three items pertaining to the perceived level of knowledge of participants, and three items on the perceived severity of COVID-19. Two additional items from the WHO survey on COVID-19 status of participants, specifically whether they have been infected with the novel coronavirus or someone in their immediate social circle has been infected, will also be recorded. ${ }^{38}$

The eight-item eHEALS Scale will be used to assess the electronic sources and channels of information-seeking behaviour related to the COVID-19 outbreak and exposure to and seeking of different types of health-related information, perceived credibility, accuracy and usefulness of the information, and confidence in finding the accurate information. ${ }^{39}$

\section{Data collection procedure}

The CARE questionnaire will be delivered using online survey platforms or offline electronic form. Specifically, a project webpage is developed to describe the project details (https://care.hku.hk) and incorporate links to surveys in various languages developed in Qualtrics. Each participating country may use surveys in the project webpage or have the same survey set up in the corresponding institution licensed Qualtrics, or Google Form. For places where internet access is limited, we have also developed an offline electronic form in pdf format so that individually collected data can be collated into a central database. All these platforms will be tested by the study team and piloted in at least ten subjects before use.

The CARE survey will be promoted via survey service providers, by snowball technique, or in social media, including Facebook, Twitter and LinkedIn. Participants will be reached by survey service providers, local community centres, social media or/and snowball sampling, whichever is/are feasible in the country. In addition, in places where there are lockdowns, university students will be contacted to complete the survey and to disseminate the survey to their family members. Each participating country shall opt for the option that is deemed most feasible and effective in the local setting.

In addition, we will obtain the daily number of new infections of COVID-19, cumulative cases, new deaths and cumulative deaths from the WHO Coronavirus Disease dashboard. $^{40}$

\section{Planned analysis}

Data will be exported from all electronic platforms into excel files or comma-separated values format. They will be gathered into a master database and cleaned in the $\mathrm{R}$ package. Records without any responses on all outcome measurements will be discarded. For instruments that have not been psychometrically evaluated, we will use the IRT to examine their reliability and validity, ${ }^{32}$ as well as their cross-cultural comparisons via differential item functioning assessment. ${ }^{33}$ The IRT approach is adopted instead of the conventional approaches because it is not sample-dependent.

For each country, descriptive statistics will be used to examine the participants' perceptions on the healthrelated impact of COVID-19, fear, anxiety and depression, lifestyles, COVID-19 knowledge, e-health literacy, and other measures, after weighting adjustment according to age and gender of the corresponding population. Cross-countries comparisons on outcomes will be made by univariable and multivariable regression models, with weighting adjustment by age and gender of the world population. ${ }^{41}$ The analysis will be repeated with weight adjustment of age and gender of the corresponding populations. In the multivariable analysis, we shall adjust for demographics, COVID-19 infection status, the number of infections and the number of deaths. The potential influences of incorrect responses to the validation question will be assessed by conducting the analyses with and without the inclusion of participants with incorrect responses. Should there be no substantial influences, results based on the full sample will be reported. All analyses will be conducted using the R package. A 0.05 overall level of significance will be used, with Holm's procedure to account for multiplicity.

\section{DISCUSSION}

As the COVID-19 pandemic continues to affect different countries in varying degrees, its health impact is yet to be comprehensively examined. As an important first step in addressing the health-related impacts of the pandemic, in terms of mental health and lifestyle, the international CARE survey will explore the experiences of people globally and will study the health-related impact and the community's needs in preparation for a pandemic in details.

To provide further information on the impact of and preparedness for the pandemic, the CARE survey findings can inform interventions that target any identified health-related areas or needs and can be developed to prepare for a future pandemic or public health calamity. Given that COVID-19 has affected countries globally, this is an important study for international collaborative research on the pandemic's global impact.

If the CARE survey reaches or exceeds its target of 500 participants per participating country, with over 30 countries involved, it will represent one of the largest international health surveys conducted on the topic to date. 
Moreover, the large sample size will enable significant subgroup analyses and could help inform international and local policy and strategy with regard to COVID-19 and future pandemic response. On the community front, people would be better informed of the challenges they may face during a pandemic. On the policy front, organisations and policy-makers would be informed on what can be strategies in order to help the community-dwelling individuals to cope with the pandemic.

The limitations of the study are important to note. With voluntary participation, it may be susceptible to self-selection bias. While the cross-sectional design could provide essential data for cross-country comparison, it may also limit the extent to which the results might be comparable for countries at vastly different stages of pandemic or pandemic response during survey administration. Additionally, health-related impacts and the perceived preparation needs of the pandemic may change across the various stages of the pandemic in different communities. While the international component of the study may allow for comparison between communities, the changes within communities may not be detected.

In summary, the CARE study will represent an extensive international health survey conducted worldwide. The study will shed new light on the health-related impact, in terms of physical, mental and lifestyle, of the COVID-19 pandemic. It will provide new insights into the challenges faced by the global population during the pandemic. Consequently, the findings will help us better understand the health impact and expectations of the Chinese population compared with other countries. The implications of these findings will be to map these health-related impacts, experiences, expectations against existing public health or healthcare supply to enable policymakers, healthcare providers and researchers to identify the needs of the population to better support the health locally and globally.

\section{ETHICS AND DISSEMINATION}

The study will be conducted in accordance with the Institutional Review Board of the University of Hong Kong/ Hospital Authority Hong Kong West Cluster's ethical conduct in research, as well as the approved study protocol (UW 20-272). Ethical and R\&D approvals have been sought and obtained in each country involved in the study, where local ethics approval is needed. Participation in the study is entirely voluntary. Subjects will be given information online and informed consent will be taken. Only those who agree to participate in the study will start completing the survey. Respondents can withdraw from the study at any time during the survey without any consequences, all information will be kept confidential, and results will be reported in aggregate form. No personally identifiable information will be collected in order to maintain the anonymity of the participants.

The research findings will be disseminated through a strategic method. The dissemination plan will include various forms of media to reach out to a wide range of stakeholder groups and individuals at the local, national, and international levels. This will inevitably include the use of academic media (ie, peer-reviewed journal articles, national and international conference presentations), social media (ie, Facebook, Instagram, Twitter, LinkedIn), print media (ie, newspaper), broadcast media (ie, radio, television) and community engagement activities such as community forums or stakeholder meetings to widen our reach of dissemination.

\section{Patient and public involvement \\ No patient involved.}

Acknowledgements We would like to thank Miss Bobo Chan for her tremendous help in the administration of the project. We also thank Dr Evelyn Balsells, Miss Ningjing Chen, Ms Chanchan Wu, Miss Heidi Fan, Mr Jayson Yu and Miss Sujin Nam for their support in translation involved in this project.

Collaborators CARE Group:Khalid M Alabdulwahhab; Mohammad Shakil Ahmad; Nagla Abdelrahim Mohamed Ahmed; Riyaz Ahmed; Mohamed Alboraie; Meshari A Alzahrani; Anil Bilimale; Sawitree Boonpatcharanon; Samuel Byiringiro; Muhammad Kamil Che Hasan; Luisa Clausi; Walter Corzo; Faruk Adamu Dantsoho; Josephine M. De Leon; Anjanette S. De Leon; Hiba Deek; Fabio Efficace; Mayssah A El Nayal; Eduardo Ensaldo-Carrasco; Pilar Escotorin; Oluwadamilola Agnes Fadodun; Israel Opeyemi Fawole; Yong-Shian Shawn Goh; Devi Irawan; Naimah Ebrahim Khan; Binu Koirala; Ashish Krishna; Cannas Kwok; Tung Thanh Le; Daniela Giambruno Leal; Miguel Ángel Lezana-Fernández; Emery Manirambona; Leandro Cruz Mantoani; Omar Mbakeh; Fernando Meneses-González; Iman Elmahdi Mohamed; Madeleine Mukeshimana; Chinh Thi Minh Nguyen; Huong Thi Thanh Nguyen; Khanh Thi Nguyen; Son Truong Nguyen; Mohammad Said Nurumal; Bunmi Ogungbe; Akingbade Oluwadamilare; Angela CY Poon; Busayasachee Puang-Ngern; Areli Reséndiz-Rodriguez; Ceryl G Sagun; Nikhil Gauri Shankar; Kathrin Sommer; Edgardo Toro; Hanh Thi Hong Tran; Elvira L Urgel; Emmanuel Uwiringiyimana; Oscar Chavez Valdez; Tita Vanichbuncha; Naglaa Youssef.

Contributors KY-WL drafted the manuscript. DYTF is the principal investigator of the study and is responsible for conducting the study overall. DYTF, JYHW, MH, KY-WL and EPHC conceived the study. DYTF, MH, KY-WL, JYHW, EPHC, VP, PMD, WD, MT, JJL and C-CL contributed to the design of the study. All authors, including those in the CARE Group, contributed to the data acquisition, critically appraised and approved the manuscript, and assume responsibility for the contents of the manuscript.

Funding The authors have not declared a specific grant for this research from any funding agency in the public, commercial or not-for-profit sectors.

Competing interests None declared.

Patient consent for publication Not required.

Provenance and peer review Not commissioned; externally peer reviewed.

Supplemental material This content has been supplied by the author(s). It has not been vetted by BMJ Publishing Group Limited (BMJ) and may not have been peer-reviewed. Any opinions or recommendations discussed are solely those of the author(s) and are not endorsed by BMJ. BMJ disclaims all liability and responsibility arising from any reliance placed on the content. Where the content includes any translated material, BMJ does not warrant the accuracy and reliability of the translations (including but not limited to local regulations, clinical guidelines, terminology, drug names and drug dosages), and is not responsible for any error and/or omissions arising from translation and adaptation or otherwise.

Open access This is an open access article distributed in accordance with the Creative Commons Attribution Non Commercial (CC BY-NC 4.0) license, which permits others to distribute, remix, adapt, build upon this work non-commercially, and license their derivative works on different terms, provided the original work is properly cited, appropriate credit is given, any changes made indicated, and the use is non-commercial. See: http://creativecommons.org/licenses/by-nc/4.0/.

ORCID iDs

Kris Yuet-Wan Lok http://orcid.org/0000-0002-3227-0799

Daniel Yee Tak Fong http://orcid.org/0000-0001-7365-9146

Janet Y.H. Wong http://orcid.org/0000-0002-3000-4577

Mandy Ho http://orcid.org/0000-0002-4460-7969 


\section{REFERENCES}

1 Worldometers. COVID-19 coronavirus pandemic, 2020. Available: https://www.worldometers.info/coronavirus/

2 World Health Organization. Estimating mortality from COVID-19: scientific brief, 2020. Available: https://www.who.int/publications/i/ item/WHO-2019-nCoV-Sci-Brief-Mortality-2020.1 [Accessed 23 Oct 2020].

3 Moukaddam N. Fears, outbreaks, and pandemics: lessons learned, 2020. Psychiatric Times. Available: https://www.psychiatrictimes. $\mathrm{com} /$ view/fears-outbreaks-and-pandemics-lessons-learned [Accessed 18 Nov 2020].

4 World Health Organization. Overview of public health and social measures in the context of COVID-19: interim guidance, 2020. Available: https://www.who.int/publications/i/item/overview-ofpublic-health-and-social-measures-in-the-context-of-covid-19 [Accessed 23 Oct 2020]

5 World Health Organization. Coronavirus disease (COVID-19) advice for the public, 2020. Available: https://www.who.int/emergencies/ diseases/novel-coronavirus-2019/advice-for-public [Accessed 23 Oct 2020].

6 Błaszczyk-Bębenek E, Jagielski P, Bolesławska I, et al. Nutrition behaviors in Polish adults before and during COVID-19 Lockdown. Nutrients 2020;12. doi:10.3390/nu12103084. [Epub ahead of print: 10 Oct 2020]

7 Poelman MP, Gillebaart M, Schlinkert C, et al. Eating behavior and food purchases during the COVID-19 lockdown: a cross-sectional study among adults in the Netherlands. Appetite 2021;157:105002.

8 Mattioli AV, Sciomer S, Cocchi C, et al. Quarantine during COVID-19 outbreak: changes in diet and physical activity increase the risk of cardiovascular disease. Nutr Metab Cardiovasc Dis 2020;30:1409-17.

9 van der Heide A, Meinders MJ, Bloem BR, et al. The impact of the COVID-19 pandemic on psychological distress, physical activity, and symptom severity in Parkinson's disease. J Parkinsons Dis 2020;10:1355-64.

10 Wong LE, Hawkins JE, Langness S. Where are all the patients? Addressing Covid-19 fear to encourage sick patients to seek emergency care. NEJM Catalyst 2020.

11 Le XTT, Dang AK, Toweh J, et al. Evaluating the psychological impacts related to COVID-19 of Vietnamese people under the first nationwide partial Lockdown in Vietnam. Front Psychiatry 2020;11:824.

12 Tran BX, Nguyen HT, Le HT, et al. Impact of COVID-19 on economic well-being and quality of life of the Vietnamese during the National social distancing. Front Psychol 2020;11:565153.

13 Wang C, Chudzicka-Czupała A, Grabowski D, et al. The association between physical and mental health and face mask use during the COVID-19 pandemic: a comparison of two countries with different views and practices. Front Psychiatry 2020;11:569981.

14 Tan W, Hao F, Mclntyre RS, et al. Is returning to work during the COVID-19 pandemic stressful? A study on immediate mental health status and psychoneuroimmunity prevention measures of Chinese workforce. Brain Behav Immun 2020;87:84-92.

15 Zhang Y, Ma ZF. Impact of the COVID-19 pandemic on mental health and quality of life among local residents in Liaoning Province, China: a cross-sectional study. Int J Environ Res Public Health 2020;17. doi:10.3390/ijerph17072381. [Epub ahead of print: 3103 2020].

16 Wang C, Tee M, Roy AE, et al. The impact of COVID-19 pandemic on physical and mental health of Asians: a study of seven middleincome countries in Asia. PLoS One 2021;16:e0246824.

17 Nakazawa E, Ino H, Akabayashi A. Chronology of COVID-19 cases on the diamond Princess cruise SHIP and ethical considerations: a report from Japan. Disaster Med Public Health Prep 2020:1-8.

18 Shanahan L, Steinhoff A, Bechtiger L, et al. Emotional distress in young adults during the COVID-19 pandemic: evidence of risk and resilience from a longitudinal cohort study. Psychol Med 2020:1-10.

19 O'Connor RC, Wetherall K, Cleare S, et al. Mental health and wellbeing during the COVID-19 pandemic: longitudinal analyses of adults in the UK COVID-19 Mental Health \& Wellbeing study. Br J Psychiatry 2020:1-17.
20 Benke C, Autenrieth LK, Asselmann E, et al. Lockdown, quarantine measures, and social distancing: associations with depression, anxiety and distress at the beginning of the COVID-19 pandemic among adults from Germany. Psychiatry Res 2020;293:113462.

21 Krendl AC, Perry BL. The impact of Sheltering in place during the COVID-19 pandemic on older adults' social and mental well-being. $J$ Gerontol B Psychol Sci Soc Sci 2021;76:e53-8.

22 Probst T, Budimir S, Pieh C. Depression in and after COVID-19 lockdown in Austria and the role of stress and loneliness in lockdown: a longitudinal study. J Affect Disord 2020;277:962-3.

23 Hao F, Tan W, Jiang L, et al. Do psychiatric patients experience more psychiatric symptoms during COVID-19 pandemic and lockdown? A case-control study with service and research implications for immunopsychiatry. Brain Behav Immun 2020;87:100-6.

24 Tee CA, Salido EO, Reyes PWC, et al. Psychological state and associated factors during the 2019 coronavirus disease (COVID-19) pandemic among Filipinos with rheumatoid arthritis or systemic lupus erythematosus. Open Access Rheumatol 2020;12:215-22.

25 Tan BYQ, Chew NWS, Lee GKH, et al. Psychological impact of the COVID-19 pandemic on health care workers in Singapore. Ann Intern Med 2020;173:317-20.

26 Salazar de Pablo G, Vaquerizo-Serrano J, Catalan A, et al. Impact of coronavirus syndromes on physical and mental health of health care workers: systematic review and meta-analysis. J Affect Disord 2020;275:48-57.

27 Luo M, Guo L, Yu M, et al. The psychological and mental impact of coronavirus disease 2019 (COVID-19) on medical staff and general public - A systematic review and meta-analysis. Psychiatry Res 2020;291:113190.

28 Brooks SK, Webster RK, Smith LE, et al. The psychological impact of quarantine and how to reduce it: rapid review of the evidence. Lancet 2020;395:912-20.

29 Chong YY, Cheng HY, Chan HYL, et al. COVID-19 pandemic, infodemic and the role of eHealth literacy. Int J Nurs Stud 2020;108:103644.

30 Conn KM, CH M, Sellers LM. When less is more in boosting survey response Rates*. Social Science Quarterly 2019;100:1445-58.

31 Wild D, Grove A, Martin M, et al. Principles of good practice for the translation and cultural adaptation process for patient-reported outcomes (pro) measures: report of the ISPOR Task force for translation and cultural adaptation. Value Health 2005;8:94-104.

32 Tsutsumi A, Iwata N, Watanabe N, et al. Application of item response theory to achieve cross-cultural comparability of occupational stress measurement. Int J Methods Psychiatr Res 2009;18:58-67.

33 Li S, Fong DYT, Wong JYH, et al. Nonrestorative sleep scale: reliable and valid for the Chinese population. Qual Life Res 2019;28:1685-92.

34 Champion VL, Skinner CS, Menon U, et al. A breast cancer fear scale: psychometric development. J Health Psychol 2004;9:753-62.

35 Kroenke K, Spitzer RL, Williams JBW, et al. An ultra-brief screening scale for anxiety and depression: the PHQ-4. Psychosomatics 2009;50:613-21.

36 Tarrant M, KM W, Dodgson JE. An investigation of the factors contributing to the early cessation of breastfeeding in Hong Kong. American Public Health Association's 138th Annual Meeting - Social Justice: A Public Health Imperative; November, 2010, Denver, CO, 2010.

37 Rivenbark JG, Copeland WE, Davisson EK, et al. Perceived social status and mental health among young adolescents: evidence from census data to cellphones. Dev Psychol 2019;55:574-85.

38 World Health Organization. Monitoring knowledge, risk perceptions, preventive behaviours and trust to inform pandemic outbreak repsonse, 2020. Available: https://www.euro.who.int/_data/assets/ pdf file/0007/436705/COVID-19-survey-tool-and-guidance.pdf

$39 \mathrm{MaZ}$, Wu M. The psychometric properties of the Chinese eHealth literacy scale (C-eHEALS) in a Chinese rural population: crosssectional validation study. J Med Internet Res 2019;21:e15720.

40 World Health Organization. WHO coronavirus disease (COVID-19) Dashboard, 2020. Available: https://covid19.who.int/table

41 United Nations. World population prospects 2019, 2020. Available: https://population.un.org/wpp/DataQuery/ 